\title{
Influence of Compost, Humic Acid and Effective Microorganisms on Organic Production of Red Cabbage
}

\author{
S. A. Abd E-Aziz ${ }^{*}$ and M. Z. E-Shinawy ${ }^{* *}$ \\ *Central Lab of Organic Agriculture, Horticulture Research \\ Institute, Agricultural Research Centre and ${ }^{* *}$ Horticulture \\ Department, Faculty of Agriculture, Ain Shams University, \\ Cairo, Egypt.
}

\begin{abstract}
A $\mathrm{N}$ EXPERIMENT was conducted during the two successive $A$ winter seasons of 2012/2013 and 2013/2014, in the experimental farm of Arid Land Agricultural Research and Service Center (ALARC), Fac. of Agriculture, Ain Shams University, Cairo, Egypt. The present work aims to study the influence of humic acid and effective microorganisms (EM) on organic production of red cabbage (Lisa $F_{1}$ Hybrid) under sandy soil conditions. The rates of compost (100 and $150 \%$ as recommended dose of nitrogen) with and without additions of humic acid and effective microorganisms (EM) individually or in combinations, were investigated comparing to recommended dose of NPK as mineral fertilizer (control) on growth, yield and quality of red cabbage. The results showed that the rates of 100 and $150 \%$ compost + humic acid + EM and $150 \%$ compost + EM only gave significantly superior in growth, yield and some quality of red cabbage compared to recommended dose of NPK as mineral fertilizer. It is recommended that good organic production of red cabbage in sandy soil can be performed successfully using rate of $150 \%$ of compost plus EM with or without addition of humic acid or using $100 \%$ compost plus humic acid and EM.
\end{abstract}

Keywords: Red cabbage, Organic production, Compost, Humic acid, Effective microorganisms (EM).

Red cabbage is usually consumed fresh as an ingredient of coleslaws and mixed salads. Cabbage, a member of cruciferae, is one of the vegetables and an important source of food globally, it is a rich source of vitamin A and C (FAO, 2000). Consumption of cruciferous vegetables, such as cabbage, is known to reduce the risk of several cancers, especially lung, colon, breast, ovarian and bladder cancer. Research also reveals that crucifers provide significant cardiovascular benefits (Beecher, 1994).

Organic farming products are becoming very necessary in today's world to manage ecosystem health and to impart related human health benefits, world over there is growing demand for organic products. The organic areas in the whole world reached to 37.5 million hectares, whereas the cultivated organic area in Egypt is about 82000 hectares (FiBL and IFOAM, 2014). In the near future, the export of vegetable will focus on the organic production. Organic 
fertilizers can therefore be used to reduce the amount of toxic compounds (such as nitrates) produced from mineral fertilizers particularly in the fresh vegetables and improving the quality of leafy vegetables produced as well as human health (Worthington, 2001 and Mahmoud et al., 2009). Fundamentals of organic farming technology is producing healthy fruits without the use of chemical fertilizers, synthetic hormones and pesticides, the means of achieving this target are through the soil becomes fertile by using compost and microorganisms to improve soil helping crops grow healthy and sturdy (Wakui, 2009).

The use of compost as organic fertilizer allows improvement in soil fertility, in addition to being excellent soil conditioner, improving their physical, chemical and biological, such as water retention, aggregation, porosity, increased the cation exchange capacity, increased fertility and improve life soil microbial, however, the value compound fertilizer depends on the material used as raw material (Miyasaka et al., 1997, Ahmad et al., 2008 and Fiorentino \& Fagnano, 2011).

Humic acid has an essential role in agricultural processes. It increases cation exchange capacity and enhances soil fertility (Chen and Aviad, 1990), converting the mineral elements into forms available for plants (Stevenson, 1994). Humic substances lead to a greater uptake of nutrients into the plant root and through the cell membrane (Tipping, 2002). Humic substances may possibly enhance the uptake of minerals through the stimulation of microbiological activity (Mayhew, 2004).

The effective microorganisms (EM) inoculants are liquid microbial concoctions containing yeasts, actinomycetes, lactic acid and photosynthetic bacteria (Daly and Stewart, 1999). Most of the species in EM inoculants are heterotrophic and require organic sources of carbon and nitrogen for their nutrition. Therefore, EM inoculation has been more effective when applied in combination with organic materials to provide both carbon and nitrogen (Yamada and $\mathrm{Xu}, 2000$ ). The microorganisms contained in the concoctions reportedly produce plant hormones, beneficial bioactive substances and antioxidants while solubilizing nutrients (Higa and Parr, 1994). The application of EM increases soil organisms those are beneficial for plant growth, resulting in more rapid mineralization of organic matter, suppression of soil-borne pathogens and increased crop yield and quality (Asia-Pacific Natural Network, 1995). Other studies have shown that inoculation of the agroecosystem with EM leads to an improvement in soil and crop quality in addition to higher crop yields (Higa and Parr, 1994, Li and Ni, 1995, Yan and Xu, 2002, Javaid, 2006, Khaliq et al., 2006, Chantal et al., 2010, Javaid \& Mahmood, 2010, Javaid, 2011 and Ncube et al., 2011). Therefore this work aimed to evaluate the ability of enhancing organic production of red cabbage by humic acid and effective microorganisms in presence of compost under sandy soil conditions.

\section{Materials and Methods}

The experiment was carried out in the experimental farm of the Arid Land Agricultural Research Center (ALARC), Faculty of Agriculture, Ain Shams

Egypt. J. Hort. Vol. 42, No. 2 (2015) 
University, Egypt, during the two successive seasons of 2012/2013 and 2013/2014. The red cabbage (Brassica oleracea var. capitata rubra) seeds (Lisa $F_{1}$ Hybrid) were sown in the nursery on 11 and 7 November in the first and second seasons, respectively. The seeds were placed in the seedling trays, which were filled with peat moss and vermiculite $1: 1(\mathrm{v}: \mathrm{v})$. Then the plants were transplanted in sandy soil on 13 and 11 of December in the first and second seasons, respectively. The experimental trial was conducted in washed sand in cement plots $\left(1 \mathrm{~m}^{2}\right)$. Each plot included 6 plants in two lines, the distance between plants was $30 \mathrm{~cm}$, were irrigated by drip irrigation. Emitter discharge rate was $4 \mathrm{~L} / \mathrm{hr}$. Chemical analyses of the experimental soil are in Table 1.

TABLE 1. Chemical analyses of the experimental soil.

\begin{tabular}{|c|c|c|c|c|c|c|c|c|c|}
\hline \multirow{2}{*}{$\begin{array}{l}\text { pH } \\
1: 5\end{array}$} & \multirow{2}{*}{$\begin{array}{c}\text { EC } 1: 10 \\
\mathrm{dS} / \mathrm{m}\end{array}$} & \multicolumn{4}{|c|}{ Cations meq/L } & \multicolumn{4}{|c|}{ Anions meq/L } \\
\hline & & $\mathrm{Ca}^{++}$ & $\mathbf{M g}^{++}$ & $\mathrm{Na}^{+}$ & $\mathbf{K}^{+}$ & $\mathrm{Cos}^{-}$ & $\mathrm{HCO}_{3}^{-}$ & $\mathrm{Cl}^{-}$ & $\mathrm{SO}_{4}=$ \\
\hline 7.58 & 0.82 & 1.55 & 0.32 & 1.36 & 0.58 & - & 1.63 & 1.81 & 1.14 \\
\hline
\end{tabular}

Two rates of compost 100 and $150 \%$ from nitrogen recommended dose in sandy soil at $70 \mathrm{~kg} \mathrm{~N} /$ feddan (fed $=0.4 \mathrm{ha}$ ) with and without addition of humic acid and effective microorganisms (EM) individually or in combinations were investigated for organic production of red cabbage comparing to conventional production (recommended dose of NPK) by mineral fertilizer (control). The compost was produced by Arid Land Agricultural Research and Service Center, Faculty of Agriculture, Ain Shams University. The chemical analyses of the used compost are illustrated in Table 2. The mineral fertilizers of NPK were applied according to Ministry of Agriculture and Land Reclamation (2009) as follow: 70 $\mathrm{kg} \mathrm{N} / \mathrm{fed}$ as $340 \mathrm{~kg}$ ammonium sulphate $(20.5 \% \mathrm{~N}), 45 \mathrm{~kg} \mathrm{P}_{2} \mathrm{O}_{5} / \mathrm{fed}$ as $290 \mathrm{~kg}$ calcium super phosphate $\left(15.5 \% \mathrm{P}_{2} \mathrm{O}_{5}\right)$ and $48 \mathrm{~kg} \mathrm{~K} 2 \mathrm{O} / \mathrm{fed}$ as $100 \mathrm{~kg}$ potassium sulphate $\left(48 \% \mathrm{~K}_{2} \mathrm{O}\right)$. Calcium super phosphate was added as one dose during soil preparation, whereas ammonium sulphate and potassium sulphate were added at three equal portions, during soil preparation, after 3 and 6 weeks from transplanting. Each quantity of compost (6.25 and 9.38 ton/fed as 100 and $150 \%$ of $\mathrm{N}$ recommended dose) were added as one dose during soil preparation. The diluted humic acid was prepared by adding $3 \mathrm{ml} / \mathrm{L}$ water. EM was produced by Ministry of Agriculture on liquid microbial concoction containing yeasts, actinomycetes, lactic acid and photosynthetic bacteria. The diluted EM was prepared by adding 2 ml concentrated EM with $2 \mathrm{ml}$ molas / L water without chlorine for 24 hours (Yadav, 2002). The diluted solutions of humic acid and EM individually or in combinations were applied to the soil surface beside plants at 4 times after 2, 4, 6 and 8 weeks from transplanting as 2 liters for plot.

TABLE 2. Chemical analyses of the compost.

\begin{tabular}{|c|c|c|c|c|c|c|c|c|c|c|c|}
\hline \multirow{2}{*}{$\begin{array}{l}\text { pH } \\
1: 5\end{array}$} & \multirow{2}{*}{$\begin{array}{c}\text { EC 1:10 } \\
\text { dS/m }\end{array}$} & \multirow{2}{*}{$\begin{array}{l}\text { O.M } \\
(\%)\end{array}$} & \multicolumn{5}{|c|}{ Macro elements (\%) } & \multicolumn{4}{|c|}{ Micro elements (ppm) } \\
\hline & & & $\mathbf{N}$ & $\mathbf{P}$ & K & $\mathrm{Ca}$ & Mg & $\mathrm{Fe}$ & $\mathbf{Z n}$ & Mn & $\mathbf{C u}$ \\
\hline 7.74 & 5.32 & 32.40 & 1.12 & 0.71 & 1.40 & 1.26 & 0.63 & 3165 & 96 & 238 & 146 \\
\hline
\end{tabular}

Egypt. J. Hort. Vol. 42, No. 2 (2015) 
The Experimental Treatments

- Recommended dose of NPK as mineral fertilizers (RDMF) (control).

- $100 \%$ compost $(100 \%$ C)

- $100 \%$ compost + humic acid (100\% C + HA)

- $100 \%$ compost $+\mathrm{EM}(100 \% \mathrm{C}+\mathrm{EM})$

- $100 \%$ compost + humic acid + EM (100\% C +HA + EM)

- $150 \%$ compost $(150 \% \mathrm{C})$

- $150 \%$ compost + humic acid $(150 \% \mathrm{C}+\mathrm{HA})$

- $150 \%$ compost + EM $(150 \% \mathrm{C}+\mathrm{EM})$

- $150 \%$ compost + humic acid + EM (150\% C + HA + EM)

The experimental treatments were arranged in a completely randomized block design, with three replicates for each treatment, each replicate included 6 plants.

After 90 days fromtransplanting, the plants were harvested and total yield was recorded for each plot. Three plants were randomly chosen from each plot to measure fresh and dry shoot weight, stem diameter (under the first bottom leaf directly), stemlength, outer leaf number, surround, length and weight of head. Also, head firmness and percentage of total soluble solids (TSS) were measured by using Pressure Tester and Digital Refractometer, respectively. Vitamin C in red cabbage heads was determined as described in FAO (1980). As well as, anthocyanin content was determined using spectrophotometer according to Geza et al. (1984). Red cabbage head content of macronutrient $(\mathrm{N}, \mathrm{P}, \mathrm{K}$ and $\mathrm{Ca}$ ), micronutrient ( $\mathrm{Fe}, \mathrm{Zn}, \mathrm{Mn}$ and $\mathrm{Cu}$ ), and nitrate were determined in dry matter of head. Total nitrogen was determined by Kjeldahl method according to the procedure described by FAO (1980). Phosphorus content was determined using spectrophotometer according to Watanabe and Olsen (1965). The $\mathrm{K}, \mathrm{Ca}, \mathrm{Fe}, \mathrm{Zn}, \mathrm{Mn}$ and $\mathrm{Cu}$ were determined spectrometrically using Phillips Unicum Atomic Absorption Spectrometer as described by Chapman and Pratt (1961). Nitrate content was determined using Brucine method reported by Holty and Potworowski (1972).

Data were statistically analyzed by the analysis of variance using one way ANOVA according to Snedecor and Cochran (1980) with using SAS package. Comparis on of treatment means was done using Tukey test at significance level 0.05.

\section{Results and Discussion}

\section{Plant growth}

Data in Table 3 showed that $150 \%$ compost + HA + EM treatment gave the highest fresh and dry weights of red cabbage plants compared to other treatments. The treatments of $100 \%$ compost + HA + EM and $150 \%$ compost + EM ranked the second order with superior on recommended dose of mineral fertilizer treatment (control). The treatments of recommended dose, 100\% compost + EM and $150 \%$ compost + HA came in the third order. The lowest fresh and dry weights of plants Egypt. J. Hort. Vol. 42, No. 2 (2015) 
were obtained by $100 \%$ compost treatment only in both seasons. This trend was true in stem diameter property. On the other hand, there weren't significant differences among the treatments of recommended dose of mineral fertilizer, $100 \%$ compost + $\mathrm{HA}+\mathrm{EM}$ and $150 \%$ compost $+\mathrm{EM}$. Concerning properties of stemlength and outer leaf number, there were no significant differences among all treatments. Generally, application of $150 \%$ compost $+\mathrm{EM}$ with or without $\mathrm{HA}$ and $100 \%$ compost $+\mathrm{HA}+$ EM significantly increased plant growth for red cabbage plants compared to the recommended dose of mineral fertilizer treatment. These effects could be a result of using high rate of compost with HA due to improving physical, chemical and biological properties of the soil (Chen \& Avaid, 1990, Miyasaka et al., 1997, Mayhew, 2004, Ahmad et al., 2008 and Fiorentino \& Fagnano, 2011). As well as, EM inoculation has more effect when applied in combination with organic materials to provide both carbon and nitrogen which are important for their nutrition (Yamada and $\mathrm{Xu}, 2000)$. Effective microorganisms produce plant hormones and beneficial bioactive substances that led to increase the plants growth (Higa \& Parr, 1994, Chantal et al., 2010 and Javaid, 2011).

TABLE 3. Effect of compost, HA and EM on fresh and dry weight of shoot (F \& DWS ), stem diameter, stem length and outer leaf number of red cabbage plants during 2012/2013 and 2013/2014 seasons.

\begin{tabular}{|c|c|c|c|c|c|}
\hline Treatments & $\begin{array}{c}\text { FWS } \\
\text { kg/plant }\end{array}$ & $\begin{array}{c}\text { DWS } \\
\text { g/plant }\end{array}$ & $\begin{array}{c}\text { Stem } \\
\text { diameter } \\
(\mathrm{cm})\end{array}$ & $\begin{array}{c}\text { Stem } \\
\text { length } \\
(\mathrm{cm})\end{array}$ & $\begin{array}{c}\text { outer } \\
\text { leaf No }\end{array}$ \\
\hline & \multicolumn{5}{|c|}{ First season } \\
\hline RDMF (control) & $1.18 \mathrm{c}$ & $113 \mathrm{c}$ & $3.38 \mathrm{bc}$ & $4.13 \mathrm{a}$ & $22.67 \mathrm{a}$ \\
\hline $100 \%$ compost & $0.91 \mathrm{e}$ & $87 \mathrm{e}$ & $2.80 \mathrm{e}$ & $3.97 \mathrm{a}$ & $22.33 \mathrm{a}$ \\
\hline $100 \%$ compost $+\mathrm{HA}$ & $1.08 \mathrm{~d}$ & $101 d$ & $3.12 \mathrm{~d}$ & $4.07 \mathrm{a}$ & $22.67 \mathrm{a}$ \\
\hline $100 \%$ compost + EM & $1.13 \mathrm{~cd}$ & $108 \mathrm{~cd}$ & $3.23 \mathrm{~cd}$ & $4.00 \mathrm{a}$ & $22.00 \mathrm{a}$ \\
\hline $100 \%$ compost $+\mathrm{HA}+\mathrm{EM}$ & $1.31 \mathrm{~b}$ & $124 \mathrm{~b}$ & $3.43 b$ & $4.27 \mathrm{a}$ & $22.67 \mathrm{a}$ \\
\hline $150 \%$ compost & $1.06 \mathrm{~d}$ & $103 d$ & $3.17 \mathrm{~d}$ & $4.07 \mathrm{a}$ & $21.67 \mathrm{a}$ \\
\hline $150 \%$ compost $+\mathrm{HA}$ & $1.17 \mathrm{c}$ & $114 \mathrm{c}$ & $3.17 \mathrm{~d}$ & $4.17 \mathrm{a}$ & $21.67 \mathrm{a}$ \\
\hline $150 \%$ compost + EM & $1.27 \mathrm{~b}$ & $123 b$ & $3.55 \mathrm{~b}$ & $4.28 \mathrm{a}$ & $23.67 \mathrm{a}$ \\
\hline \multirow[t]{2}{*}{$150 \%$ compost $+\mathrm{HA}+\mathrm{EM}$} & $1.42 \mathrm{a}$ & $135 \mathrm{a}$ & $3.86 \mathrm{a}$ & $4.18 \mathrm{a}$ & $23.33 \mathrm{a}$ \\
\hline & \multicolumn{5}{|c|}{ Second season } \\
\hline RDMF (control) & $1.26 b c$ & $115 \mathrm{c}$ & $3.68 \mathrm{bc}$ & $4.72 \mathrm{a}$ & $23.40 \mathrm{a}$ \\
\hline $100 \%$ compost & $0.97 \mathrm{~d}$ & $88 \mathrm{e}$ & $3.02 \mathrm{f}$ & $4.73 \mathrm{a}$ & $22.91 \mathrm{a}$ \\
\hline $100 \%$ compost $+\mathrm{HA}$ & $1.16 \mathrm{c}$ & $102 d$ & $3.23 \mathrm{ef}$ & $4.74 \mathrm{a}$ & $23.26 \mathrm{a}$ \\
\hline $100 \%$ compost + EM & $1.20 \mathrm{c}$ & $108 \mathrm{~d}$ & $3.55 \mathrm{~cd}$ & $4.56 \mathrm{a}$ & $23.19 \mathrm{a}$ \\
\hline $100 \%$ compost $+\mathrm{HA}+\mathrm{EM}$ & $1.37 \mathrm{ab}$ & $126 \mathrm{~b}$ & $3.75 \mathrm{bc}$ & $4.83 \mathrm{a}$ & $24.07 \mathrm{a}$ \\
\hline $150 \%$ compost & $1.22 \mathrm{bc}$ & $104 d$ & $3.42 \mathrm{de}$ & $4.64 \mathrm{a}$ & $23.22 \mathrm{a}$ \\
\hline $150 \%$ compost $+\mathrm{HA}$ & $1.26 b c$ & $115 \mathrm{c}$ & $3.56 \mathrm{~cd}$ & $4.75 \mathrm{a}$ & $23.72 \mathrm{a}$ \\
\hline $150 \%$ compost + EM & $1.46 \mathrm{a}$ & $124 b$ & $3.86 \mathrm{~b}$ & $4.86 \mathrm{a}$ & $24.42 \mathrm{a}$ \\
\hline $150 \%$ compost $+\mathrm{HA}+\mathrm{EM}$ & $1.51 \mathrm{a}$ & $137 \mathrm{a}$ & $4.15 \mathrm{a}$ & $4.87 \mathrm{a}$ & $24.75 \mathrm{~A}$ \\
\hline
\end{tabular}

Means in same column followed by similar letters are not statistically different at 0.05 level according to Tukey test.

RDMF $=$ Recommended Dose Of Mineral Fertilizer

$\mathrm{HA}=$ Humic Acid

$\mathrm{EM}=$ Effective Microorganisms 


\section{Macro and micronutrients}

Macronutrients content of red cabbage plants is shown in Table 4. The highest concentrations of N, P and $\mathrm{K}$ were produced by $150 \%$ compost plus EM with or without HA treatments followed by the recommended dose of mineral fertilizer and $100 \%$ compost $+\mathrm{HA}+\mathrm{EM}$ treatments (Table 4). On the other hand, the lowest percentages of $\mathrm{N}, \mathrm{P}$ and $\mathrm{K}$ in red cabbage plants were found with $100 \%$ compost without adding HA or EM treatment. While $150 \%$ compost with HA and EM individually or in combinations, recommended dose of mineral fertilizer and $100 \%$ compost $+\mathrm{HA}+\mathrm{EM}$ treatments resulted in the highest percentages of $\mathrm{Ca}$ in red cabbage plants. On the contrary, $100 \%$ compost only gave the lowest $\mathrm{Ca} \%$.

TABLE 4. Effect of compost, HA and EM on macronutrient percent of red cabbage plants during 2012/2013 and 2013/2014 seasons.

\begin{tabular}{|c|c|c|c|c|}
\hline Treatments & $\mathrm{N}(\%)$ & $\mathbf{P}(\%)$ & $\mathrm{K}(\%)$ & $\mathrm{Ca}(\%)$ \\
\hline & \multicolumn{4}{|c|}{ First season } \\
\hline RDMF (control) & $3.42 b$ & $0.445 \mathrm{~cd}$ & $2.45 \mathrm{bc}$ & $1.61 \mathrm{ab}$ \\
\hline $100 \%$ compost & $2.23 \mathrm{f}$ & $0.248 \mathrm{~g}$ & $1.32 \mathrm{f}$ & $0.86 \mathrm{f}$ \\
\hline $100 \%$ compost $+\mathrm{HA}$ & $2.50 \mathrm{e}$ & $0.334 \mathrm{f}$ & $1.67 \mathrm{e}$ & $1.18 \mathrm{e}$ \\
\hline $100 \%$ compost $+\mathrm{EM}$ & $3.08 \mathrm{c}$ & $0.384 \mathrm{e}$ & $2.03 \mathrm{~d}$ & $1.29 \mathrm{~d}$ \\
\hline $100 \%$ compost $+\mathrm{HA}+\mathrm{EM}$ & $3.41 \mathrm{~b}$ & $0.435 \mathrm{~cd}$ & $2.45 \mathrm{bc}$ & $1.59 \mathrm{~b}$ \\
\hline $150 \%$ compost & $2.87 \mathrm{~d}$ & $0.422 \mathrm{~d}$ & $2.29 \mathrm{c}$ & $1.44 \mathrm{c}$ \\
\hline $150 \%$ compost $+\mathrm{HA}$ & $3.23 \mathrm{c}$ & $0.453 \mathrm{c}$ & $2.35 \mathrm{c}$ & $1.63 \mathrm{ab}$ \\
\hline $150 \%$ compost $+\mathrm{EM}$ & $3.68 \mathrm{a}$ & $0.516 b$ & $2.61 \mathrm{ab}$ & $1.66 \mathrm{ab}$ \\
\hline \multirow[t]{2}{*}{$150 \%$ compost $+\mathrm{HA}+\mathrm{EM}$} & $3.73 \mathrm{a}$ & $0.553 \mathrm{a}$ & $2.78 \mathrm{a}$ & $1.68 \mathrm{a}$ \\
\hline & \multicolumn{4}{|c|}{ Second season } \\
\hline RDMF (control) & $3.55 \mathrm{~b}$ & $0.457 b$ & $2.52 b$ & $1.63 b$ \\
\hline $100 \%$ compost & $2.30 \mathrm{f}$ & $0.255 \mathrm{f}$ & $1.37 \mathrm{e}$ & $0.88 \mathrm{f}$ \\
\hline $100 \%$ compost $+\mathrm{HA}$ & $2.59 \mathrm{e}$ & $0.343 \mathrm{e}$ & $1.73 \mathrm{~d}$ & $1.20 \mathrm{e}$ \\
\hline $100 \%$ compost $+\mathrm{EM}$ & $3.20 \mathrm{~d}$ & $0.395 \mathrm{~d}$ & $2.14 \mathrm{c}$ & $1.41 \mathrm{~d}$ \\
\hline $100 \%$ compost $+\mathrm{HA}+\mathrm{EM}$ & $3.55 \mathrm{~b}$ & $0.451 b c$ & $2.58 \mathrm{~b}$ & $1.62 \mathrm{bc}$ \\
\hline $150 \%$ compost & $3.10 \mathrm{~d}$ & $0.424 \mathrm{c}$ & $2.46 b$ & $1.55 \mathrm{c}$ \\
\hline $150 \%$ compost $+\mathrm{HA}$ & $3.38 \mathrm{c}$ & $0.466 \mathrm{~b}$ & $2.52 b$ & $1.67 \mathrm{ab}$ \\
\hline $150 \%$ compost $+\mathrm{EM}$ & $3.85 \mathrm{a}$ & $0.514 \mathrm{a}$ & $2.81 \mathrm{a}$ & $1.70 \mathrm{ab}$ \\
\hline $150 \%$ compost $+\mathrm{HA}+\mathrm{EM}$ & $3.91 \mathrm{a}$ & $0.535 \mathrm{a}$ & $2.99 \mathrm{a}$ & $1.72 \mathrm{a}$ \\
\hline
\end{tabular}

Means in same column followed by similar letters are not statistically different at 0.05 level according to Tukey test.

RDMF $=$ Recommended Dose Of Mineral Fertilizer

$\mathrm{HA}=$ Humic Acid

$\mathrm{EM}=$ Effective Microorganisms

Egypt. J. Hort. Vol. 42, No. 2 (2015) 
Data in Table 5 indicated that applying $150 \%$ compost with or without HA or $\mathrm{EM}$ increased micronutrients content ( $\mathrm{Fe}, \mathrm{Zn}, \mathrm{Mn}$ and $\mathrm{Cu}$ ). Conversely, applying $100 \%$ compost without HA or EM decreased Fe and $\mathrm{Zn}$ content. Whereas, there were no significant differences among $100 \%$ compost without HA or EM treatments and recommended dose of mineral fertilizer treatment in plants content of $\mathrm{Mn}$ and $\mathrm{Cu}$. These effects approximately were true in the two seasons. These results might be due to the beneficial effects of compost which increase cation exchange capacity of sandy soil to maintain nutrients in available form for plants (Miyasaka et al., 1997, Ahmad et al., 2008 and Fiorentino \& Fagnano, 2011). Moreover, positive effects of humic acid and EM may increase the stimulation of microbiological activity and enhance the nutrients uptake by plant roots through solubilizing of nutrients (Stevenson, 1994, Higa \& Parr, 1994, Tipping, 2002, Mayhew, 2004 and Ncube et al., 2011).

TABLE 5. Effect of compost, HA and on micronutrient concentration (ppm) of red cabbage plants during 2012/2013 and 2013/2014 seasons.

\begin{tabular}{|c|c|c|c|c|}
\hline Treatments & $\mathrm{Fe}(\mathrm{ppm})$ & Zn (ppm) & Mn (ppm) & $\mathrm{Cu}(\mathbf{p p m})$ \\
\hline \multicolumn{5}{|c|}{ First season } \\
\hline RDMF (control) & $111.87 \mathrm{c}$ & $29.50 \mathrm{c}$ & $26.67 \mathrm{~b}$ & $10.37 \mathrm{c}$ \\
\hline $100 \%$ compost & $68.50 \mathrm{e}$ & $20.43 \mathrm{~d}$ & $22.43 b$ & $8.20 \mathrm{c}$ \\
\hline $100 \%$ compost $+\mathrm{HA}$ & $85.67 \mathrm{~d}$ & $22.53 d$ & $25.43 b$ & $9.37 \mathrm{c}$ \\
\hline $100 \%$ compost $+\mathrm{EM}$ & $95.77 d$ & $23.37 d$ & $25.40 \mathrm{~b}$ & $9.03 c$ \\
\hline $100 \%$ compost $+\mathrm{HA}+\mathrm{EM}$ & $109.87 \mathrm{c}$ & $24.57 \mathrm{~d}$ & $26.10 \mathrm{~b}$ & $9.67 \mathrm{c}$ \\
\hline $150 \%$ compost & $111.87 \mathrm{~b}$ & $30.50 \mathrm{bc}$ & $36.27 \mathrm{a}$ & $13.00 \mathrm{~b}$ \\
\hline $150 \%$ compost $+\mathrm{HA}$ & $140.77 \mathrm{a}$ & $35.20 \mathrm{a}$ & $38.27 \mathrm{a}$ & $15.23 \mathrm{ab}$ \\
\hline $150 \%$ compost $+\mathrm{EM}$ & $140.10 \mathrm{a}$ & $34.17 \mathrm{ab}$ & $37.00 \mathrm{a}$ & $15.07 \mathrm{ab}$ \\
\hline $150 \%$ compost $+\mathrm{HA}+\mathrm{EM}$ & $143.37 \mathrm{a}$ & $36.43 \mathrm{a}$ & $38.93 a$ & $16.40 \mathrm{a}$ \\
\hline \multicolumn{5}{|c|}{ Second season } \\
\hline RDMF (control) & $118.94 \mathrm{bc}$ & $30.34 \mathrm{~cd}$ & $27.96 \mathrm{~b}$ & $10.74 \mathrm{~cd}$ \\
\hline $100 \%$ compost & $68.88 \mathrm{e}$ & $21.01 \mathrm{f}$ & $22.85 \mathrm{c}$ & $8.50 \mathrm{~d}$ \\
\hline $100 \%$ compost $+\mathrm{HA}$ & $88.09 \mathrm{~d}$ & $23.17 \mathrm{ef}$ & $26.66 b c$ & $9.71 d$ \\
\hline $100 \%$ compost $+\mathrm{EM}$ & $98.48 \mathrm{~d}$ & $24.03 \mathrm{ef}$ & $26.63 b c$ & $9.36 \mathrm{~d}$ \\
\hline $100 \%$ compost $+\mathrm{HA}+\mathrm{EM}$ & $113.18 \mathrm{c}$ & $27.09 \mathrm{de}$ & $27.36 \mathrm{~b}$ & $11.02 \mathrm{~cd}$ \\
\hline $150 \%$ compost & $128.77 b$ & $31.97 \mathrm{bc}$ & $38.02 \mathrm{a}$ & $13.54 \mathrm{bc}$ \\
\hline $150 \%$ compost $+\mathrm{HA}$ & $146.78 \mathrm{a}$ & $35.82 \mathrm{ab}$ & $40.12 \mathrm{a}$ & $15.90 \mathrm{ab}$ \\
\hline $150 \%$ compost $+\mathrm{EM}$ & $147.62 \mathrm{a}$ & $36.90 \mathrm{a}$ & $38.79 a$ & $15.73 \mathrm{ab}$ \\
\hline $150 \%$ compost $+\mathrm{HA}+\mathrm{EM}$ & $151.07 \mathrm{a}$ & $38.19 \mathrm{a}$ & $40.81 \mathrm{a}$ & $17.12 \mathrm{a}$ \\
\hline
\end{tabular}

Means in same column followed by similar letters are not statistically different at 0.05 level according to Tukey test.

RDMF $=$ Recommended Dose of Mineral Fertilizer

HA = Humic Acid

$\mathrm{EM}=$ Effective Microorganisms 


\section{Yield and head quality}

Data in Tables 6 and 7 revealed that $150 \%$ compost $+\mathrm{HA}+\mathrm{EM}$ treatments gave the highest values of total yield, weight, surround and length of head compared to other treatments. The treatments of $100 \%$ compost $+\mathrm{HA}+\mathrm{EM}$ and $150 \%$ compost $+\mathrm{EM}$ came in the second order that were superior compared to the recommended dose of mineral fertilizer treatment. The lowest values of total yield, weight, surround and length of head were obtained by $100 \%$ compost without HA or EM treatments in both seas ons. These effects might be due to that most of microorganism species in EM inoculants are heterotrophic and require organic sources of carbon and nitrogen for their nutrition. Therefore, EM inoculation has more effect when applied in combination with organic materials (Yamada and $\mathrm{Xu}, 2000$ ).

TABLE 6. Effect of compost, HA and EM on yield, weight, surround and length of red cabbage heads during 2012/2013 and 2013/2014 seasons.

\begin{tabular}{|l|c|c|c|c|}
\hline Treatments & $\begin{array}{c}\text { yield } \\
\mathbf{k g} / \mathbf{m}^{2}\end{array}$ & $\begin{array}{c}\text { Head weight } \\
\mathbf{k g}\end{array}$ & $\begin{array}{c}\text { Head } \\
\text { surround cm }\end{array}$ & Head length cm \\
\hline & \multicolumn{4}{|c|}{ First season } \\
\hline RDMF (control) & $4.50 \mathrm{~cd}$ & $0.747 \mathrm{c}$ & $39.33 \mathrm{bc}$ & $18.50 \mathrm{bcd}$ \\
\hline $100 \%$ compost & $3.47 \mathrm{f}$ & $0.580 \mathrm{e}$ & $35.33 \mathrm{~d}$ & $16.00 \mathrm{f}$ \\
\hline $100 \%$ compost $+\mathrm{HA}$ & $4.12 \mathrm{e}$ & $0.687 \mathrm{~d}$ & $37.33 \mathrm{~cd}$ & $17.17 \mathrm{e}$ \\
\hline $100 \%$ compost $+\mathrm{EM}$ & $4.31 \mathrm{de}$ & $0.720 \mathrm{~cd}$ & $38.67 \mathrm{~cd}$ & $18.00 \mathrm{de}$ \\
\hline $100 \%$ compost $+\mathrm{HA}+\mathrm{EM}$ & $5.01 \mathrm{~b}$ & $0.843 \mathrm{ab}$ & $41.33 \mathrm{ab}$ & $19.00 \mathrm{abc}$ \\
\hline $150 \%$ compost & $4.11 \mathrm{e}$ & $0.680 \mathrm{~d}$ & $37.67 \mathrm{~cd}$ & $18.17 \mathrm{~cd}$ \\
\hline $150 \%$ compost $+\mathrm{HA}$ & $4.53 \mathrm{c}$ & $0.760 \mathrm{c}$ & $39.67 \mathrm{bc}$ & $18.50 \mathrm{bcd}$ \\
\hline $150 \%$ compost $+\mathrm{EM}$ & $5.00 \mathrm{~b}$ & $0.840 \mathrm{~b}$ & $41.33 \mathrm{ab}$ & $19.33 \mathrm{ab}$ \\
\hline $150 \%$ compost $+\mathrm{HA}+\mathrm{EM}$ & $5.27 \mathrm{a}$ & $0.883 \mathrm{a}$ & $43.33 \mathrm{a}$ & $19.67 \mathrm{a}$ \\
\hline & & Second season & \\
\hline RDMF control) & $4.72 \mathrm{c}$ & $0.836 \mathrm{c}$ & $41.60 \mathrm{c}$ & $18.90 \mathrm{bc}$ \\
\hline $100 \%$ compost & $3.58 \mathrm{f}$ & $0.629 \mathrm{e}$ & $37.00 \mathrm{e}$ & $16.20 \mathrm{f}$ \\
\hline $100 \%$ compost $+\mathrm{HA}$ & $4.30 \mathrm{e}$ & $0.753 \mathrm{~d}$ & $38.96 \mathrm{~d}$ & $17.47 \mathrm{e}$ \\
\hline $100 \%$ compost $+\mathrm{EM}$ & $4.52 \mathrm{~d}$ & $0.783 \mathrm{~d}$ & $40.42 \mathrm{~cd}$ & $18.43 \mathrm{~d}$ \\
\hline $100 \%$ compost $+\mathrm{HA}+\mathrm{EM}$ & $5.27 \mathrm{~b}$ & $0.905 \mathrm{~b}$ & $43.37 \mathrm{~b}$ & $19.28 \mathrm{~b}$ \\
\hline $150 \%$ compost & $4.32 \mathrm{e}$ & $0.746 \mathrm{~d}$ & $39.63 \mathrm{~d}$ & $18.57 \mathrm{~cd}$ \\
\hline $150 \%$ compost $+\mathrm{HA}$ & $4.73 \mathrm{c}$ & $0.834 \mathrm{c}$ & $41.73 \mathrm{c}$ & $18.86 \mathrm{c}$ \\
\hline $150 \%$ compost $+\mathrm{EM}$ & $5.35 \mathrm{~b}$ & $0.933 \mathrm{ab}$ & $44.06 \mathrm{ab}$ & $19.85 \mathrm{a}$ \\
\hline $150 \%$ compost $+\mathrm{HA}+\mathrm{EM}$ & $5.55 \mathrm{a}$ & $0.961 \mathrm{a}$ & $45.117 \mathrm{a}$ & $20.00 \mathrm{a}$ \\
\hline
\end{tabular}

Means in same column followed by similar letters are not statistically different at 0.05 level according to Tukey test.

RDMF $=$ Recommended Dose of Mineral Fertilizer

$\mathrm{HA}=$ Humic acid

$\mathrm{EM}=$ Effective Microorganisms

Egypt. J. Hort. Vol. 42, No. 2 (2015) 
TABLE 7. Effect of compost, $\mathrm{HA}$ and EM on firmness, TSS, vitamin C, anthocyanin and nitrate of red cabbage heads during 2012/2013 and 2013/2014 seasons.

\begin{tabular}{|c|c|c|c|c|c|}
\hline \multirow{2}{*}{ Treatments } & Firmness & TSS & Vit. C & $\begin{array}{c}\text { Anthocyani } \\
n\end{array}$ & $\mathrm{NO}_{3}$ \\
\hline & $\mathrm{Kg} / \mathrm{cm}^{2}$ & $\%$ & $\mathrm{mg} / 100 \mathrm{~g}$ & $\%$ & $\%$ \\
\hline & \multicolumn{5}{|c|}{ First season } \\
\hline RDMF (control) & $6.50 \mathrm{~b}$ & $5.20 \mathrm{bc}$ & $33.27 \mathrm{~cd}$ & $0.308 \mathrm{de}$ & $0.340 \mathrm{a}$ \\
\hline $100 \%$ compost & $5.27 \mathrm{~d}$ & $3.77 \mathrm{e}$ & $32.07 \mathrm{~g}$ & $0.296 \mathrm{~h}$ & $0.062 \mathrm{~d}$ \\
\hline $100 \%$ compost $+\mathrm{HA}$ & $5.53 \mathrm{~cd}$ & $4.07 \mathrm{e}$ & $32.47 \mathrm{fg}$ & $0.300 \mathrm{gh}$ & $0.067 \mathrm{~cd}$ \\
\hline $100 \%$ compost $+\mathrm{EM}$ & $6.13 b c$ & $4.63 d$ & $32.57 \mathrm{ef}$ & $0.302 \mathrm{fg}$ & $0.072 \mathrm{~cd}$ \\
\hline $100 \%$ compost $+\mathrm{HA}+\mathrm{EM}$ & $6.70 \mathrm{ab}$ & $5.60 \mathrm{ab}$ & $33.67 b c$ & $0.312 \mathrm{~cd}$ & $0.079 \mathrm{~cd}$ \\
\hline $150 \%$ compost & $5.77 \mathrm{~cd}$ & $4.87 \mathrm{~cd}$ & $32.93 \mathrm{de}$ & $0.304 \mathrm{ef}$ & $0.091 \mathrm{c}$ \\
\hline $150 \%$ compost $+\mathrm{HA}$ & $6.13 b c$ & $5.13 \mathrm{bcd}$ & $33.80 \mathrm{bc}$ & $0.313 b c$ & $0.117 b$ \\
\hline $150 \%$ compost $+\mathrm{EM}$ & $6.70 \mathrm{ab}$ & $5.73 a$ & $34.23 \mathrm{a}$ & $0.317 \mathrm{ab}$ & $0.123 b$ \\
\hline \multirow[t]{2}{*}{$150 \%$ compost $+\mathrm{HA}+\mathrm{EM}$} & $7.23 \mathrm{a}$ & $5.93 a$ & $34.63 \mathrm{a}$ & $0.321 \mathrm{a}$ & $0.132 b$ \\
\hline & \multicolumn{5}{|c|}{ Second season } \\
\hline RDMF (control) & $6.32 b c$ & $5.10 \mathrm{~cd}$ & $32.99 \mathrm{c}$ & $0.304 b$ & $0.350 \mathrm{a}$ \\
\hline $100 \%$ compost & $5.05 \mathrm{e}$ & $3.66 \mathrm{~g}$ & $31.67 \mathrm{f}$ & $0.291 \mathrm{e}$ & $0.062 \mathrm{~d}$ \\
\hline $100 \%$ compost $+\mathrm{HA}$ & $5.40 \mathrm{de}$ & $3.95 \mathrm{~g}$ & $32.16 \mathrm{e}$ & $0.295 \mathrm{de}$ & $0.068 \mathrm{~d}$ \\
\hline $100 \%$ compost $+\mathrm{EM}$ & $5.96 \mathrm{c}$ & $4.53 \mathrm{f}$ & $32.40 \mathrm{de}$ & $0.296 \mathrm{~cd}$ & $0.073 \mathrm{~cd}$ \\
\hline $100 \%$ compost $+\mathrm{HA}+\mathrm{EM}$ & $6.48 b$ & $5.34 \mathrm{bc}$ & $33.53 b$ & $0.307 \mathrm{~b}$ & $0.083 \mathrm{~cd}$ \\
\hline $150 \%$ compost & $5.52 \mathrm{~d}$ & $4.73 \mathrm{ef}$ & $32.63 d$ & $0.299 \mathrm{c}$ & $0.092 \mathrm{c}$ \\
\hline $150 \%$ compost $+\mathrm{HA}$ & $6.05 \mathrm{c}$ & 4.99de & $33.49 b$ & $0.308 b$ & $0.118 b$ \\
\hline $150 \%$ compost $+\mathrm{EM}$ & $6.58 \mathrm{ab}$ & $5.64 \mathrm{ab}$ & $34.00 \mathrm{a}$ & $0.314 \mathrm{a}$ & $0.125 b$ \\
\hline $150 \%$ compost $+\mathrm{HA}+\mathrm{EM}$ & $6.94 a$ & $5.77 \mathrm{a}$ & $34.26 \mathrm{a}$ & $0.316 \mathrm{a}$ & $0.130 \mathrm{~b}$ \\
\hline
\end{tabular}

Means in same column followed by similar letters are not statistically different at 0.05 level according to Tukey test.

RDMF $=$ Recommended Dose of Mineral Fertilizer

$\mathrm{HA}=$ Humic Acid

$\mathrm{EM}=$ Effective Microorganisms

Application of $150 \%$ compost + EM with or without HA and $100 \%$ compost $+\mathrm{HA}+\mathrm{EM}$ increased head firmness of cabbage, while $100 \%$ compost with or without HA and $150 \%$ compost only decreased head firmness, whereas other treatments were mediated. This effect could be resulted from the reduction of calcium concentration in the plant tissues that treated by $100 \%$ compost with or without HA and $150 \%$ compost only (Table 4) where this element in plant tis sue is located in the middle lamella, which give the strength to the cell wall, thus lead to an increase in head firmness as was reported by Marschner (1995). The same trend approximately was true with TSS, vitamin $C$ and anthocyanin content in red cabbage heads in the two seasons. As increasing the rate of compost to $150 \%$ with HA and EM individually or in combinations increased TSS, vitamin $\mathrm{C}$ and anthocyanin content in red cabbage heads.

Egypt. J. Hort. Vol. 42, No. 2 (2015) 
Generally, increasing of compost rate to $150 \%$ with addition of HA and EM significantly increased plant growth, yield and quality for red cabbage. The positive influences of this treatment may be due to the availability of nutrients in the soil, which lead to an increase in vegetative growth of plants (Table 3), that may result in accumulation of more carbohydrates enhancing yield and head quality. These results were supported by Li \& Ni (1995), Asia-Pacific Natural Network (1995) on natural agriculture, Yan \& Xu (2002) on peanut, Javaid (2006) on pea, Chantal et al. (2010) on cabbage, Javaid \& Mahmood (2010) on soybean and Ncube et al. (2011) on tomato.

Concerning nitrate content in red cabbage heads in both seasons, all compost with or without $\mathrm{HA}$ and $\mathrm{EM}$ treatments reduced $\mathrm{NO}_{3}$ content of red cabbage heads significantly comparing with the recommended dose of mineral fertilizer treatment. This explains that applying organic fertilizer decrease nitrate accumulation in vegetables as were found by El-Shinawy et al. (1999) and Abou-El-Hassan \& Desoky (2013) on lettuce, Worthington (2001) on vegetables, Mahmoud et al. (2009) on cucumber and Shehata et al. (2010) on celery.

\section{Conclusions}

It could be concluded that organic production of red cabbage in sandy soil can be enhanced successfully by applying $150 \%$ of compost as recommended dose of nitrogen + EM with or without humic acid or using 100\% compost + humic acid and EM to produce good yield, quality and healthy of red cabbage.

\section{References}

Abou-El-Hassan, S. and Desoky, A.H. (2013) Effect of compost and compost tea on organic production of head lettuce. J. Appl. Sci. Res., 9 (11), 5650-5655.

Ahmad, R., Shehzad, S.M., Khalid, A., Arshad, M. and Mahmood, M.H. (2008) Growth and yield response of wheat and maize to nitrogen and L tryptophan enriched compost. Pak. J. Bot., 39(2), 541-549.

Asia-Pacific Natural Agriculture Network (1995) EM application manual for APNAN countries. M. Shintani (ed). Asia-Pacific Natural Agriculture Network Bangkok, Thailand. http://www.agriton.nl/apnanman.html.

Beecher, C. (1994) Cancer preventive properties of varieties of Brassica oleracea a review. Am. J. Clin. Nutr., 59, 1166 S.

Chantal, K., Xiaohou, S. Weimu, W. and IroOngor, B.T. (2010) Effects of effective microorganisms on yield and quality of vegetable cabbage comparatively to nitrogen and phosphorus fertilizers. Pak. J. Nutr., 9 (11), 1039-1042.

Chapman, H.D. and Pratt, P.F. (1961) Methods of Analysis for Soil, Plant and Water, Division of Agric. Sci., Calif. Univ.

Egypt. J. Hort. Vol. 42, No. 2 (2015) 
Chen, Y. and Aviad, T. (1990) Effects of humic substances on plant growth. In: McCarthy P, Calpp CE, Malcolm RL. Bloom, Readings.ASA and SSSA, Madison, WI. pp. 161-186.

Daly, M.J. and Stewart, D.C.P. (1999) Influence of effective microorganisms on vegetable production and carbon mineralization a preliminary investigation. Sust. Agr., 14, 15-28.

El-Shinawy, M.Z., Abd-Elmoniem, E.M. and Abou-Hadid, A.F. (1999) The use of organic manure for lettuce plants grown under NFT conditions. Acta Hort., 491, 315318.

FAO (Food and Agriculture Organization) (1980) Soil and Plant Analysis. Soils Bulletin, 38 (2) 250.

FAO (Food and Agriculture Organization) (2000) Statistical database food and Agricultural Organization of the United Nations, Rome, Italy.

FiBL and IFOAM (2014) The World of Organic Agriculture, Statistics and Emerging Trends.

Fiorentino, N. and M. Fagnano, (2011) Soil fertilization with composted solid waste: short term effects on lettuce production and mineral $\mathrm{N}$ availability. Geophysical Research Abstracts, Vol. 13, p. 10520.

Geza, H., Parsons, G.F. and Maattick, L.R. (1984) Physiological and biochemical events during development and maturation of grape berries. Am. J. Enol. Vitic., 35 (4), 220-227.

Higa, T. and Parr, J.F. (1994) Beneficial and effective microorganisms for a sustainable agriculture and environment.International Nature Farming Research Center, Atami, Japan. http://www.agriton.nl/higa.html.

Holty, J.G. and Potworowski, H.S. (1972) Brucine analysis for high nitrate Concentrations. Enviro. Sci. Tech., 8 (6), 835- 837.

Javaid, A. (2006) Foliar application of effective microorganisms on pea as an alternative fertilizer. Agronomy for Sustainable Development, 26, 257-262.

Javaid, A. (2011) Effects of biofertilizers combined with different soil amendments on potted rice plants. Chil. J. Agr. Res., 71 (1), 157-163.

Javaid, A. and Mahmood, N. (2010) Growth and nodulation response of soybean to biofertilizers. Pakistan J. of Botany, 42, 863-871.

Khaliq, A., Abbasi, M.K. and Hussain, T. (2006) Effect of integrated use of organic and inorganic nutrient sources with effective microorganisms (EM) on seed cotton yield in Pakistan. Bioresource Technology, 97, 967-972. 
Li, W. and Ni, Y. (1995) Research and application of EM (effective microorganisms). Chin. J. Ecol., 14, 58-62.

Mahmoud, E.K., Abd EL-Kader, N., Robin, P., Akkal-Corfini, N. and Abd ElRahman, L. (2009) Effects of different organic and inorganic fertilizers on Cucumber Yield and Some Soil Properties. World J. Agric. Sci., 5 (4), 408-414.

Marschner, H. (1995) "Mineral Nutrition of Higher Plants" $2^{\text {nd }}$ ed., Academic press. New York. pp. 243-244.

Mayhew, L. (2004) Humic substances in biological agriculture [Online].Available at www.acresusa.com/toolbox/reprints/Jan04_Humic\%20Substances.pdf.

Ministry of Agriculture and Land Reclamation (2009) Symptoms of nutrient deficiency on some field and horticultural crops. Soils, Water and Enviro. Res. Inst., Agric. Res. Center.

Miyasaka, S., Nakamura, Y. and Okamoto, H. (1997) Yield and nutrient absorption by lettuce by liming and fertilization mineral and organic soil. Brazilian Horticulture, $\mathbf{8}$ (2), 6-9.

Ncube, L., Mnkeni, P.N.S. and Brutsch, M.O. (2011) Agronomic suitability of effective micro-organisms for tomato production. Afr. J. Agric. Res., 6(3), 650-654.

Shehata, S.M., Abdel-Azem, H.S., Abou El-Yazied, A. and El-Gizawy, A.M. (2010) Interactive effect of mineral nitrogen and biofertilization on the growth, chemical composition and yield of celeriac plant. European Journal of Scientific Research, 47 (2), 248-255.

Snedecor, G.W. and Cochran, (1980) "Statistical Methods", $6^{\text {th }}$ ed., Iowa state university press, Ames., Iowa, U.S.A.

Stevenson, F.J. (1994) Humus Chemistry: Genesis, composition, reactions, $2^{\text {nd }}$ edition, John Wiley and Sons, Inc, New York.

Tipping, E. (2002) Cation binding by humic substances. Cambridge University Press, Cambridge, U.K.

Wakui, Y. (2009) Organic farming technology in Japan. The Japan Agricultural Exchange Council. Nikken Align Bldg. Nishi-Kamata, Ota-ku, Tokyo, Japan.

Watanabe, F.S. and Olsen, S.R. (1965) Test of an ascorbic acid method for determining phosphorus in water and $\mathrm{Na}_{\mathrm{HCO}_{3}}$ extracts from soil. Soil Sci. Soc. Amer. Proc., 29, $677-678$.

Worthington, V. (2001) Nutritional quality of organic versus conventional fruits, vegetables and grains. J. Alternative Complent. Med., 7, 161-173.

Egypt. J. Hort. Vol. 42, No. 2 (2015) 
Yadav, S.P. (2002) Performance of effective microorganisms (EM) on growth and yields of selected vegetables. Nature Farming \& Environment, 1, 35-38.

Yamada, K. and XU, H.L. (2000) Properties and applications of an organic fertilizer inoculated with effective microorganisms. Crop Prod., 3, 255-268.

Yan, P.S. and Xu, H.L. (2002) Influence of EM Bokashi on nodulation, physiological characters and yield of peanut in nature farming fields. J. Sus. Agric.,19, 105-112.

(Received 8/10/2014; accepted 10/2/2015)

تأثير الكمبوست وحمض الهيوميك والكائنات الحية الاقيقة النشطة على الإنتاج العضوى للكرنب الاحمر

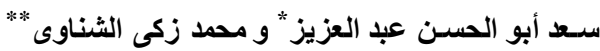

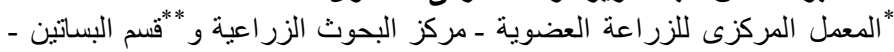

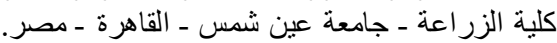

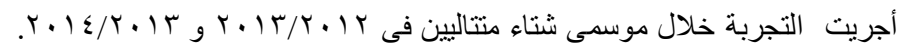

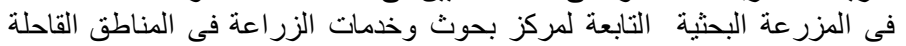

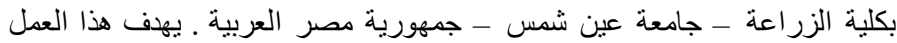

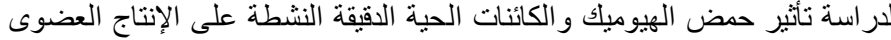
للكرنب الاحمر (هجين ليز ا) تحت ظروف التربة الرملية.

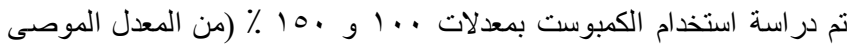

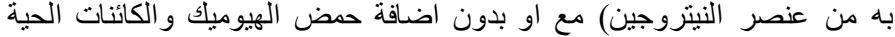

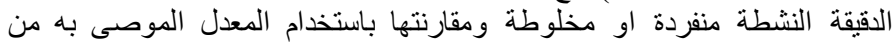

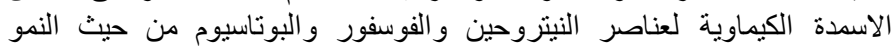

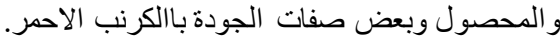

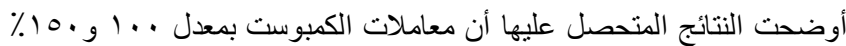

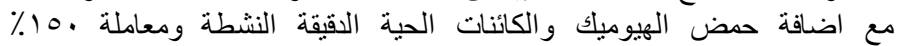

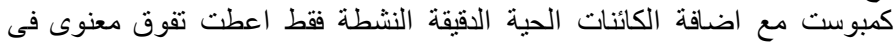

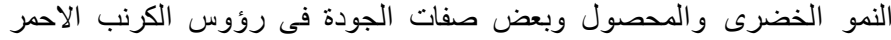
مقارنة بمعاملة المعدل الموصى به من السماد المعدنى (المقارنة).

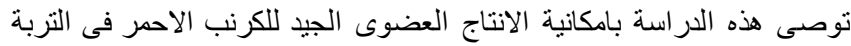

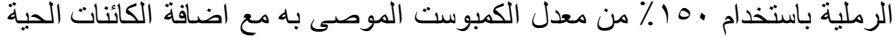

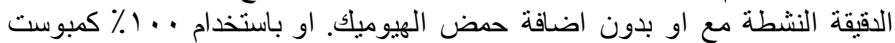
مع اضافة حمض الهيوميك وال الكائنات الحية القيقة النيقة النشطة. 